\title{
Density of states
}

\author{
Kurt Langfeld ${ }^{* \dagger}$ \\ Department for the Mathematical Sciences, University of Liverpool, Liverpool, L69 7ZL, UK \\ E-mail: kurt.langfeldeliverpool.ac.uk
}

\begin{abstract}
Although Monte Carlo calculations using Importance Sampling have matured into the most widely employed method for determining first principle results in QCD, they spectacularly fail for theories with a sign problem or for which certain rare configurations play an important role. Non-Markovian Random walks, based upon iterative refinements of the density-of-states, overcome such overlap problems. I will review the Linear Logarithmic Relaxation (LLR) method and, in particular, focus onto ergodicity and exponential error suppression. Applications include the high-state Potts model, SU(2) and SU(3) Yang-Mills theories as well as a quantum field theory with a strong sign problem: QCD at finite densities of heavy quarks.
\end{abstract}

34th annual International Symposium on Lattice Field Theory

24-30 July 2016

University of Southampton, $U K$

\footnotetext{
* Speaker.

${ }^{\dagger}$ This work is supported in part by the Leverhulme Trust (grant RPG-2014-118) and STFC (grant ST/L000350/1).
} 


\section{Introduction}

Markov chain Monte-Carlo (MCMC) simulations of quantum field theories discretised on a spacetime lattice have matured into a powerful quantitative tool for ab-initio calculations. Among other non-perturbative techniques, the almost unique characteristic is the control over error margins within the approach itself.

Still nowadays, Markov chain Monte-Carlo simulations face two severe limitations

(i) due to ergodicity problems

(ii) in cases of a non-positive Gibbs factor.

Both issues are far from technical problems only, but are intrinsically connected to physics phenomenons that entirely evade rigorous results from MCMC.

Let me just mention two examples with class (i) issues: In cases of pure Yang-Mills theories with configurations falling into sectors of the so-called topological charge, even advanced methods (such as Hybrid Monte Carlo type algorithms) fail to sweep between sectors on large volumes (see [1] for a precise definition and an analysis of the issue). The second example concerns the wide range of thermal or quantum field theories with a first order phase transition: at criticality, the configuration space falls into distinct sets of configurations that are equally relevant. For the transfer between sets, MCMC needs either theory dependent information for global updates (cluster algorithms fall into this class) or needs to fall back to rather local update strategies. In the later case, configurations need to pass through regions of low probability. In fact, this probability is exponentially small with the volume $V$, i.e., $\exp \{-\sigma V\}^{1}$, and ergodicity is hampered at the practical level for relevant sized systems.

In class (ii), we e.g. find quantum field theories (QFTs) with a topological $\theta$-term or, of even bigger phenomenological relevance, QFTs with matter at finite densities or, more precisely, at finite chemical potentials. Different strategies have been explored over the decades, and I refer to the reviews at the annual Lattice Conference for a summary of recent progress $[2,3,4,5,6,7,8]$.

A more radical approach is to abandon MCMC simulations altogether and to explore instead NonMarkovian Random Walk simulations. These do not rely on an update of the configurations according to Importance Sampling with respect to a positive Gibbs factor, which makes them well suited to study class (ii) theories. One particular set-up that is particularly relevant for QFTs with class (i) and (ii) issues uses the inverse density-of-states ${ }^{2}$ as a measure for updating configuration. This measure is (semi-) positive definite by definition, and aims to generate a random walk in configuration space even in "deprived" regions with very low probabilistic measure. To my knowledge, the first of its kind is the Multicanonical Algorithm by Berg and Neuhaus [9]. The same re-weighting approach is also at the heart of the Wang-Landau approach [10] or, more recently a version adapted to continuous QFT degrees of freedom, the LLR method [11, 12], which will be the focal point of this paper (also see [13] for a recent review).

Let us now look at the definition of the density-of-states $\rho(E)$ in a QFT setting with degrees of freedom $\phi(x)$. In its simplest form, it quantifies the number of configurations for a given action

\footnotetext{
${ }^{1} \sigma$ is the tension of interfaces between pockets of different phase material at criticality.

${ }^{2} \mathrm{~A}$ precise definition will be given below.
} 

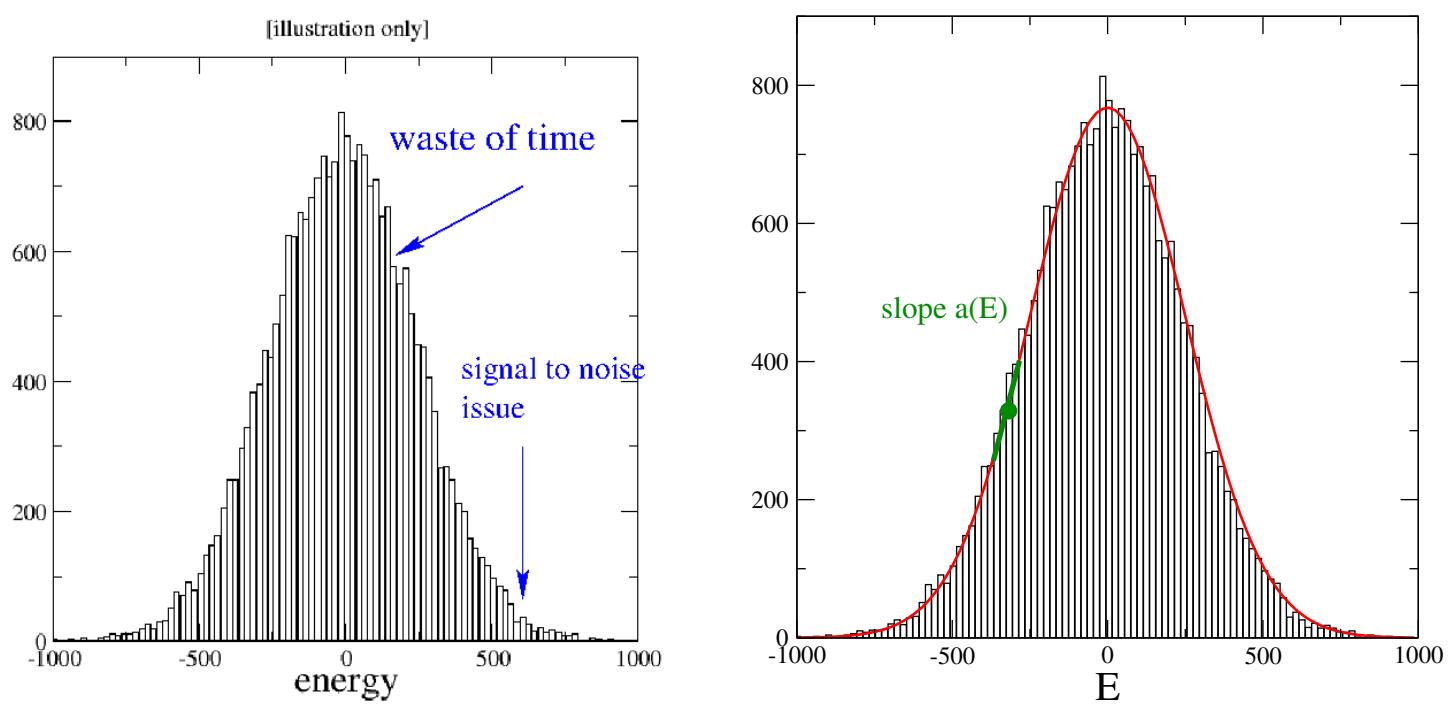

Figure 1: Illustration of the density of states $\rho(E)$

hyper-surface in configuration space:

$$
\rho(E)=\int \mathscr{D} \phi \delta(S[\phi]-E)
$$

The probabilistic weight $P(E)$ is given by the product of density and Gibbs factor, $P(E)=\rho(E) \exp \{\beta E\}$, and the partition function is recovered by performing a one-dimensional integral

$$
Z(\beta)=\int \mathscr{D} \phi \mathrm{e}^{\beta S[\phi]}=\int d E P(E)=\int d E \rho(E) \mathrm{e}^{\beta E},
$$

as can be easily verified by inserting (1.1) into (1.2). The density of states (1.1) could be straightforwardly estimated by generating configurations $\{\phi\}$ according to the plain measure, calculate the corresponding action $S[\phi]$ and accumulate statistics in a histogram. The result could look like the histogram in figure 1 , left panel. In order to obtain the statistical weight $P(E)$ with which the configuration contributes to the partition function $Z$, the density $\rho(E)$ is multiplied by the Gibbs factor. This exponentially amplifies the relevance of the configurations with large action $E$. Note, however, that we have relatively few events in the histogram at relevant values for $E$. The majority is generated around $E \approx 0$, which only marginally contribute to $Z$. Hence, we would end up with a poor signal for this amount of statistics. The target of the density-of-states method (in the LLR formulation) is to estimate the slope $a(E)$ at any chosen point $E$ (see figure 1, right panel):

$$
a(E)=\frac{d \ln \rho(E)}{d E} .
$$

Once this slope $a(E)$ is obtained, the density $\rho(E)$ can be reconstructed up to a multiplicative factor by integration:

$$
\rho(E)=\rho\left(E_{0}\right) \exp \left\{\int_{E_{0}}^{E} a\left(E^{\prime}\right) d E^{\prime}\right\} .
$$

The result of this integration is indicated by the red line in figure 1, right panel. As detailed below, crucial is that the estimate for $a(E)$ can be obtained with roughly the same statistical error 
independent of $E$ and even if $E$ is from a region of overall low weight $P(E)$. This is how the LLR approach circumvents overlap problems.

\section{The LLR method}

\subsection{Finding the LLR coefficients}

While the Multicanonical approach [9] and the Wang-Landau method [10] directly target the density $\rho(E)$, the coefficients $a(E)$ (1.3) are the primary target of the LLR method [11]. Key ingredient is the Monte-Carlo expectation value

$$
\begin{aligned}
\langle\langle W[\phi]\rangle\rangle_{E}(a) & =\frac{1}{\mathscr{N}_{E}} \int \mathscr{D} \phi \Omega_{[E, \delta E]}(S[\phi]) W[\phi] \mathrm{e}^{-a S[\phi]} . \\
\mathscr{N}_{E} & =\int \mathscr{D} \phi \Omega_{[E, \delta E]} \mathrm{e}^{-a S[\phi]},
\end{aligned}
$$

where $a$ is, for the moment, an external parameter, $W[\phi]$ is an observable, and $\Omega_{[E, \delta E]}$ is the socalled Window function. This is the only place where the $E$ dependence of $\langle\langle W[\phi]\rangle\rangle_{E}$ comes into play. Note that the double expectation value $\left\langle\langle\ldots\rangle_{E}\right.$ appears as a standard MC average and can be estimated with standard Importance Sampling methods. Historically and to maintain a close connection to the Wang-Landau method, the Window function restricts the simulation to an interval of size $\delta E$ around $E$ :

$$
\Omega_{[E, \delta E]}(S)=\left\{\begin{array}{l}
1 \text { for } E-\frac{\delta E}{2} \leq S \leq E+\frac{\delta E}{2} \\
0 \text { otherwise }
\end{array}\right.
$$

The choice of a smooth window function is of great importance if advanced MC simulation techniques such as the Hybrid Monte Carlo method are employed. In this case, the window function contributes to the MC drift force, and the algorithm enjoys the familiar high acceptance rates for configurations. Below, I will show results obtained with the choice

$$
\Omega_{[E, \delta E]}(S)=\exp \left\{-\frac{(S-E)^{2}}{\delta E^{2}}\right\} .
$$

We will be interested in the limit $\delta E \rightarrow 0$. As will become clear below, the only important constraint for potential Window functions is that $\Omega(S)$ is symmetric around $S=E$ and that it decreases sufficiently fast for $S \rightarrow \pm \infty$.

We are now prepared to study the LLR method to find the slope $a(E)$ in (1.3). To this aim, we choose $W[\phi]=S[\phi]-E$ and use the definition of $\rho$ [using $E^{\prime}$ as integration variable to avoid a clash of notation] (1.1) in $(2.1,2.2)$, which become:

$$
\begin{aligned}
\langle\langle W[\phi]\rangle\rangle_{E}(a) & =\frac{1}{\mathscr{N}_{E}} \int d E^{\prime} \Omega_{[E, \delta E]}\left(E^{\prime}\right)\left(E^{\prime}-E\right) \rho\left(E^{\prime}\right) \mathrm{e}^{-a E^{\prime}} . \\
\mathscr{N}_{E} & =\int d E \Omega_{[E, \delta E]}\left(E^{\prime}\right) \mathrm{e}^{-a E^{\prime}},
\end{aligned}
$$

The Window function confines the integration to a small, $\delta E$ sized interval around $E$. For sufficiently smooth densities $\rho\left(E^{\prime}\right)$, we might expand in leading order

$$
\ln \rho\left(E^{\prime}\right) \approx \ln \rho(E)+\frac{d \ln \rho(E)}{d E}\left(E^{\prime}-E\right)=\ln \rho(E)+a(E)\left(E^{\prime}-E\right) .
$$


Using (2.7) in (2.5,2.6), we find

$$
\begin{aligned}
\langle\langle S[\phi]-E\rangle\rangle_{E}(a) & \approx \frac{1}{N_{E}} \int d E^{\prime} \Omega_{[E, \delta E]}\left(E^{\prime}\right)\left(E^{\prime}-E\right) \exp \left\{-[a(E)-a]\left(E^{\prime}-E\right)\right\} \\
N_{E} & =\int d E \Omega_{[E, \delta E]}\left(E^{\prime}\right) \exp \left\{-[a(E)-a]\left(E^{\prime}-E\right)\right\} .
\end{aligned}
$$

Since $\Omega_{[E, \delta E]}\left(E^{\prime}\right)$ is symmetric around $E$, we find for sufficiently small $\delta E$ the key equation:

$$
\lim _{\delta E \rightarrow 0}\langle\langle S[\phi]-E\rangle\rangle_{E}(a=a(E))=0 .
$$

Remember that the expectation value $\langle\langle S[\phi]-E\rangle\rangle_{E}(a)$ is accessible by standard MC simulations for any external parameter $a$. Hence, the LLR coefficient $a(E)$ can be obtained by solving the stochastic non-linear equation:

$$
\langle\langle S[\phi]-E\rangle\rangle_{E}(a)=0 .
$$

For finite $\delta E$, it can be shown [12] that $\langle\langle S[\phi]-E\rangle\rangle_{E}(a)$ is a monotonic function and, thus, the solution is unique. A thorough study of the truncation error yields for the solution [12]:

$$
\langle\langle\Delta S[\phi]-E\rangle\rangle_{E}(a)=0 \quad \Leftrightarrow \quad a=a(E)+\mathscr{O}\left(\delta E^{2}\right) .
$$

For an alternative method to calculate the coefficients $a(E)$, we point the reader to the Functional Fit Approach [14, 15].

\subsection{Features of the LLR algorithm}

How do we solve the stochastic equation (2.9) in practice and how do we control errors for observables?

In practice, only MC estimators are available for the expectation value $\langle\langle\Delta S[\phi]-E\rangle\rangle_{E}(a)$. Let us assume that $M \mathrm{MC}$ samples are used to estimate the expectation value. Any numerical procedure for solving the stochastic equation will produce a statistical ensemble $\{a\}_{M}$ of approximate solutions. For practicability and robustness of the density-of-states method, we need to achieve two goals:

(1) The average over the ensemble $\{a\}_{M}$ needs to agree with the true solution: $\langle a\rangle=a(E)$.

(2) For sufficiently large $M$, the distribution for $\{a\}_{M}$ should be normal.

Both properties would provide us with the capability to control errors: For each $E$ and for a large enough choice $M$, we would generate $n_{B}$ approximate solutions $\bar{a}_{n}^{(M)}, n=1 \ldots n_{B}$. Because of property (2), the $n_{b}$ copies of potential solutions can be used in a standard bootstrap analysis for derived observables.

The LLR algorithms comes with a specific proposal to accomplish the above two goals. Starting point is the fix-point iteration

$$
a^{(n+1)}=a^{(n)}+c_{n} \frac{12}{\delta E^{2}}\langle\langle S[\phi]-E\rangle\rangle_{E}\left(a^{(n)}\right),
$$

with coefficients $c_{n}$ yet to be specified. For $c_{n}=1$, the above iteration is inspired by the NewtonRaphson method. The issue is that there a two sources of error: the convergence error of the iteration and the statistical uncertainty induced by replacing the expectation value $\langle\langle S[\phi]-E\rangle\rangle_{E}$ by 
MC estimators. The mathematical framework to solve this problem has been already provided by Robbins and Monro in the fifties [16]: if the coefficients satisfy

$$
\sum_{n=0}^{\infty} c_{n}=\infty \quad \text { and } \quad \sum_{n=0}^{\infty} c_{n}^{2}<\infty
$$

and if the iteration is truncated at some (large) $n=n_{R M}$, the corresponding values $a^{\left(n_{R M}\right)}$ are normal distributed with the mean coinciding with the true solution. Moreover, the limiting case, i.e., $c_{n}=$ $1 /(n+1)$, is optimal [16].

Other methods such as the Functional Fit Approach [14, 15] have been proposed to extract the slope $a(E)$ although more work to establish a systematic error control might be needed.

Once the LLR coefficients $a(E)$ have been obtained for a set of values $E$, the density $\rho(E)$ is reconstructed by integration (see (1.4)). This integration is necessarily approximate, and we call $\widetilde{\rho}(E)$ the approximation to the density of states. In practice, $\widetilde{\rho}(E)$ is affected by truncation errors, i.e., using a finite non-zero value for $\delta E$, and statistical errors for each value $E$ of the set. Key observation is, however, that the LLR approach features an exponential error suppression in the sense that [12]

$$
\rho(E)=\widetilde{\rho}(E) \exp \{\text { truncation \& statistical error }\}
$$

i.e., the relative error propagates, and $\widetilde{\rho}(E)$ approximately has a constant relative error also it might span ten thousands of orders of magnitude [12].

\subsection{Exponential error suppression at work: the SU(2) and SU(3) showcase}
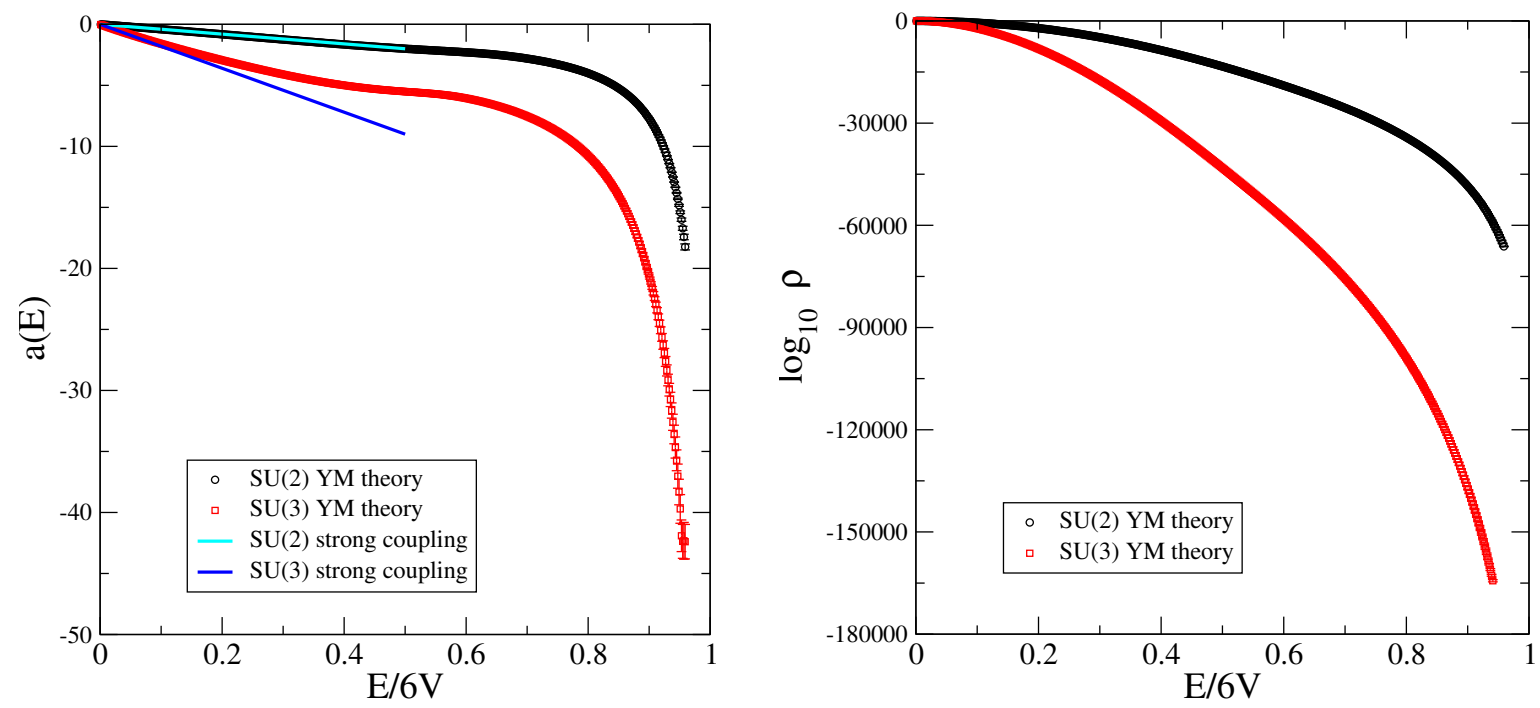

Figure 2: LLR coefficients $a(E)$ (left) and the density of states $\rho(E)$ (right) as a function of the action $E$ for a $\mathrm{SU}(2)$ and $\mathrm{SU}(3)$ lattice gauge theory on a $10^{4}$ lattice (plots from [13]).

For an illustration, I show results for the SU(2) and SU(3) pure Yang-Mills theory on a $10^{4}$ lattice. For the LLR simulations, I used the Gaussian window function (2.4) and LHMC update algorithm. 
20 (and occasionally 40) independent samples for $a(E)$ are obtained for carrying out the bootstrap error analysis.

My numerical findings for the coefficients $a(E)$ are shown in the left panel of figure 2. At small values $a(E)$, which implies small values for $E, a(E)$ can be calculated analytically using strong coupling techniques [13]. The result for the reconstructed density $\rho(E)$ for $\mathrm{SU}(2)$ and $\mathrm{SU}(3)$ can be found in the right panel of the same figure. Note that, in the SU(3) case, the density stretches over more than 150,000 orders of magnitude with almost a constant relative error over the whole range.

\subsection{Ergodicity}

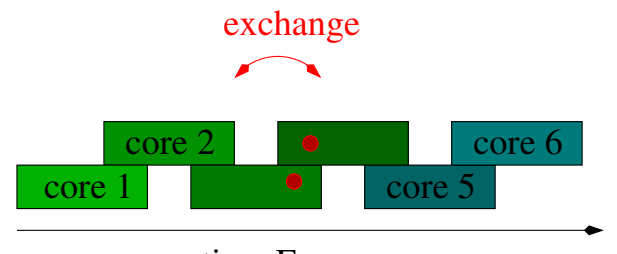

action $\mathrm{E}$

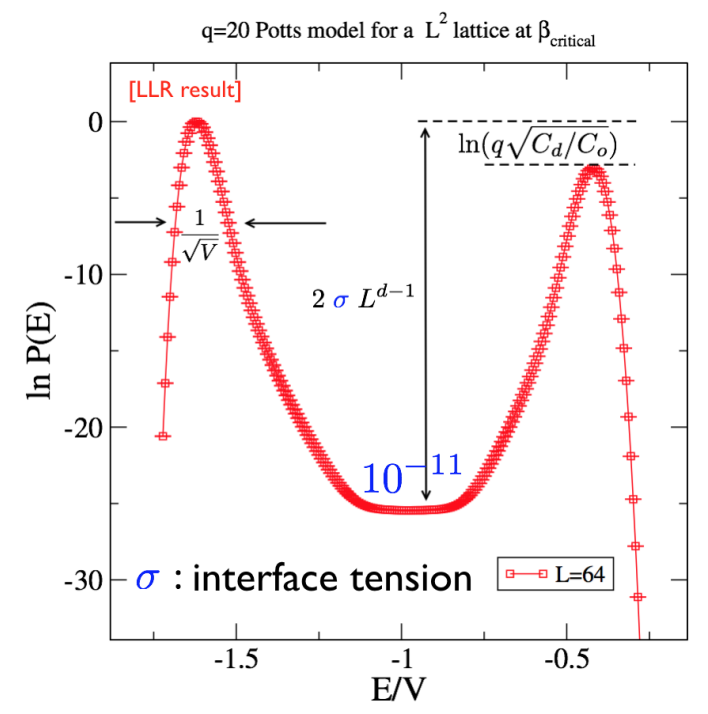

Figure 3: Left: illustration of the replica exchange method. Right: Typical double-peak structure of $P(E)$ of a high state Potts model at criticality.

Shortly after we published the first paper [11] on the LLR method, concerns were raised that the approach might suffer from ergodicity problems since the window function $\Omega_{[E, \delta E]}$ might impede the exploration of the full configuration space in particular if a step function is used for $\Omega_{[E, \delta E]}$. To resolve this issue on both a conceptional and a practical level, we proposed in [12] a generalised Replica Exchange Simulation [17]: The set of actions $E$ and the width $\delta E$ is chosen to produce overlapping action intervals (see figure 3 for an illustration). Each core of an HPC facility is now performing the LLR approach and, in particular, hosts a configuration corresponding to the action interval of the core. If two "neighbouring" cores now host configurations the action of which would be eligible for either core according to the window function (a step-function for illustration purposes here), a Monte-Carlo step decides whether both configurations are being exchanged. This approach implies that a particular configuration is not restricted to a finite action interval solving the ergodicity problem conceptually and reduces auto-correlation time significantly in practice.

Let us consider the 2-dimensional q-state Potts model on a square lattice for $q$ as high as 20 to illustrate the method. In two dimensions, many analytical results are available by virtue of the 
exact solution of the model by Baxter [18]. Our target will be the probabilistic weight $P(E)$ as a function of the action $E$ at criticality. The infinite volume critical coupling is given by $\beta_{\text {critical }}=$ $\frac{1}{2} \ln (1+\sqrt{q})$. Figure 3, right panel, summarises the expectations: the scale of the width of the individual peaks is set by $1 / \sqrt{V}$ where $V=L^{2}$ is the volume and $L$ the system size. At criticality, the relative height is determined by $q$, the degeneracy in the broken phase, and the ratio of the specific heats in ordered and disordered phase. Between the peaks, a plateau emerges, which is due to the formation of 1-dimensional interfaces. In $d$ dimension, the expectation is that the "probability floor" is given by $\exp \left\{-2 \sigma L^{d-1}\right\}$, where $\sigma$ is the interface tension.

\subsection{Ergodicity - the $q=20$ state Potts model as showcase}
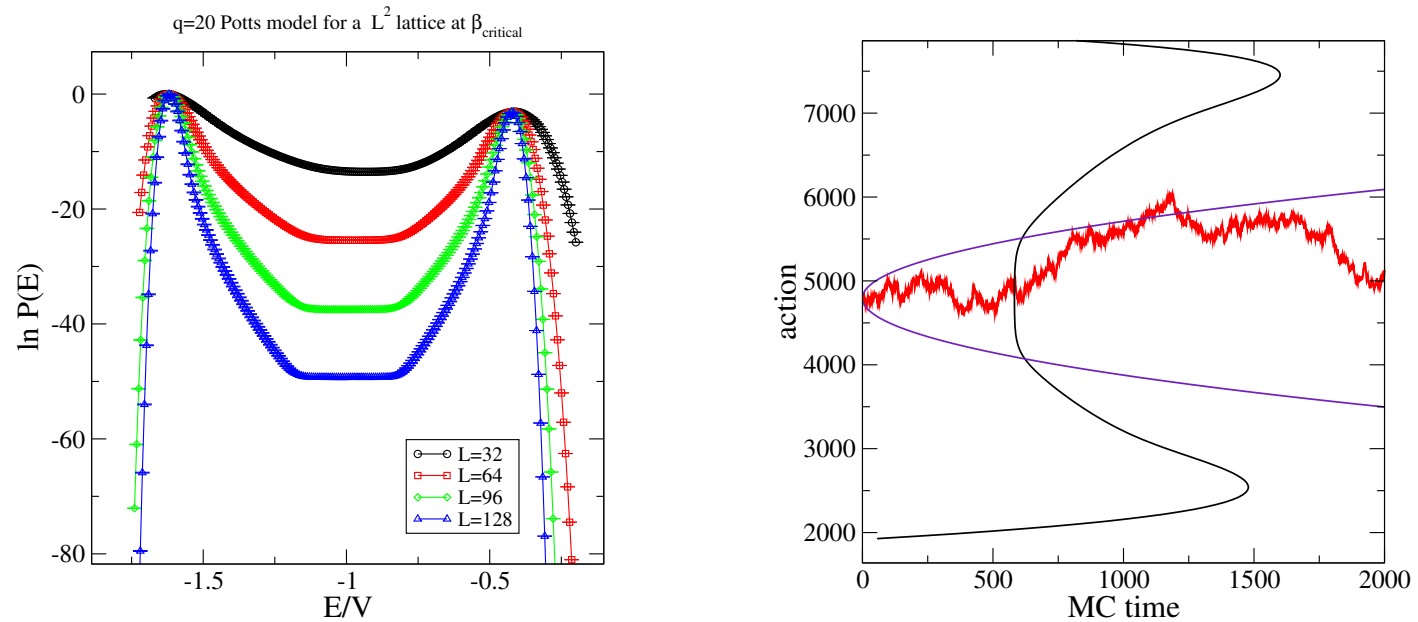

Figure 4: Left: The probabilistic weight $P(E)$ for the $2 \mathrm{~d} q=20$ Potts model for the lattice sizes $L=$ 32, 64,96, 128 using the LLR method. Right: Tracing a particular configuration throughout the simulation: shown is the action of the configuration over Robins-Monro MC simulation time $(q=20, L=64)$.

First numerical results for the case of $q=10$ exemplified the Multicanonical algorithm by Berg and Neuhaus [9], and first results for up to $q=20$ can be found in [19]. For testing the replica exchange method, I have chosen the step-function window function with action intervals that overlap by $50 \%$. This considerably alleviates the logistics of the Replica exchange step. MC spin updates are performed with standard local heat-bath method. Figure 4, left panel, shows my numerical result for the probabilistic weight $P(E)$ at criticality (in infinite volume) for several values of the lattice size $L$. Especially, the result for $L=128$ clearly shows a plateau forming due to interfaces. Also note that, on a logarithmic scale, the spacing between the plateau values linearly depends on the system size $L$. This confirms the 1-dimensional nature.

Once for a given action interval specified by $E$ the corresponding coefficient $a(E)$ has (almost) converged, the MC steps do perform a random walk in configuration space carved out by the Window function for the interval $[E, \delta E]$. If Replica steps facilitate the exchange between configurations of neighbouring action intervals, one expects that a particular configuration drifts through all of the configuration space unrestricted by action intervals. This is illustrated in figure 4 , right panel, for a $L=64$ lattice and for an interval size $\delta E=29$. The inlays show the probability distribution (black) and one standard deviation for a random walk between intervals (purple). I have monitored the 

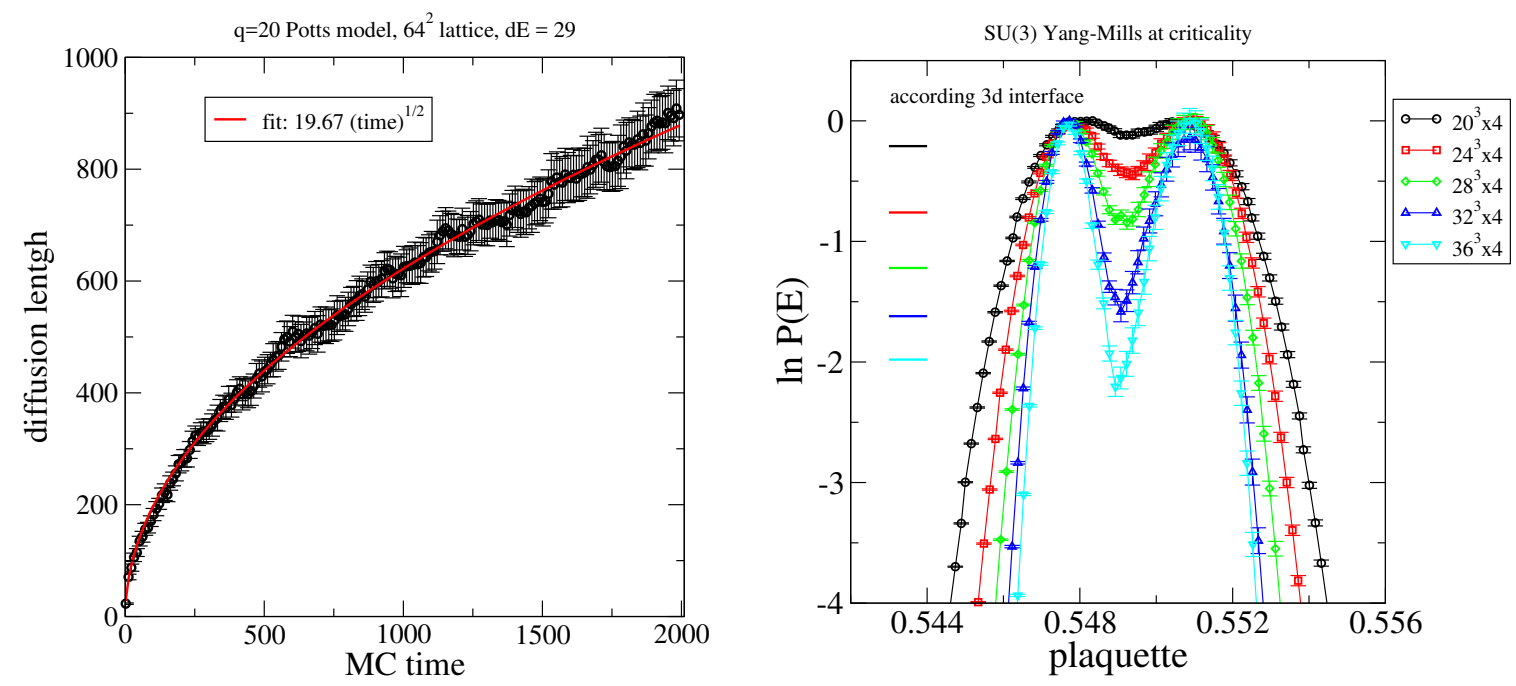

Figure 5: Left: Diffusion length as a function of the MC time in comparison to the expectation of a random walk [20-state Potts model at criticality and size $L=64$ ]. Right: $P(E)$ at criticality of the SU(3) Yang-Mills theory.

evolution of a particular configuration (red line) with an initial action from the interface regime. In this particular case, we observe that this configuration bridges 24 action intervals within $750 \mathrm{MC}$ steps. If the configurations are indeed randomly swapped by the Replica Exchange step uninhibited by any probabilistic weight, we should observe a "diffusion length" that grows with square root of the simulation time. My numerical findings in figure 5 are indeed very well fitted by this square-root law thus confirming the non-Markovian Random walk.

\subsection{Application - SU(3) Yang-Mills theory at criticality}

Let us consider the partition function of pure SU(3) Yang-Mills theory using the Wilson action, i.e.,

$$
Z(T)=\int \mathscr{D} U_{\mu} \exp \left\{\beta \sum_{x, \mu>v} \frac{1}{3} \operatorname{Re} P_{\mu v}(x)\right\},
$$

where $P_{\mu \nu}$ is the trace of the plaquette, and $U_{\mu}(x) \in \mathrm{SU}(3)$ are the gluon degrees of freedom. The theory is discretised using a lattice of size $L^{3} \times N_{t}$ with periodic boundary conditions. As usual, the temperature is given by $T=1 / N_{t} a(\beta)$. Here, we define criticality (at a finite volume), if $P(E)$ features a double-peak structure with both peaks of equal height. The critical value $\beta_{\text {crit }}$ of the Wilson coupling is obtain by fine-tuning until equal height is achieved. Any density-of-states method is ideally suited for this task since, once the density $\rho(E)$ is obtained, the probabilistic weight $P(E)=\rho(E) \exp \{\beta E\}$ is obtained for all $\beta$ values without further simulations. Once $P(E)$ is at our figure tips, a variety of thermodynamical quantities can be obtained (see the discussion of the Potts model and, in particular, figure 3, right panel): The specific heats of the confinement and the deconfinement (ordered) phase from the widths of the peaks; The latent heat from the distance between the peaks; The order-disorder interface tension from the volume scaling of the "plateau" between the peaks. In this preliminary study, I will show results for $P(E)$ for lattice sizes $N_{t}=4, L=20,24,28,32,36$. My numerical findings are shown in figure 5. Even the lattice size 
$36 \times 4$ is not large enough to clearly reveal the plateau in the action range between the peaks. For large volumes, the plateau value should scale with volume as $\ln P(E) \propto V^{-3}$. The horizontal lines at the left of the graph indicate the expectation according to the scaling law. A rough consistency could be observed, but studies with much larger volumes are needed to confirm this.

\section{The density-of-states approach for complex action systems}

The density-of-states method is not limited to the calculation of action observables. For instance in [20] the probability distribution of the Polyakov line was calculated with extreme precision for a study of 2-color QCD at finite densities of heavy quarks. Note that in the LLR approach, the only MC simulations are with respect to the density-of-states, a quantity that is (semi-) positive definite even if the variable of interest is not even real.

\subsection{The generalised density-of-states}

A wide class of theories of high phenomenological interest is spanned by quantum field theories at finite densities. The prototype partition function is given by:

$$
Z(\mu)=\int \mathscr{D} \phi \mathrm{e}^{S_{R}[\Phi](\mu)} \exp \left(i \mu S_{I}[\phi]\right)
$$

and features a complex action. In such cases, the generalised density-of-states, i.e.,

$$
P_{\beta}(s)=N \int \mathscr{D} \phi \delta\left(s-S_{I}[\phi]\right) \mathrm{e}^{S_{R}[\Phi](\mu)}
$$

is a measure for the probability of finding a particular value $s$ for the complex part of the action. An early attempt to estimate $P_{\beta}(s)$ was proposed by Gocksch [21] . If $P(s)$ is known, the partition function is recovered by a 1-dimensional Fourier transform:

$$
Z(\mu)=\int d s P_{\beta}(s) \exp \{i \mu s\}
$$

Not that $s$ is an extensive quantity. An indicative probability distribution and the resulting partition function is given by

$$
P_{\beta}(s)=\exp \left\{-\frac{s^{2}}{V}\right\}, \quad Z=\int d s \mathrm{e}^{-s^{2} / V} \exp \{i \mu s\} \propto \exp \left\{-\frac{u^{2}}{4} V\right\} .
$$

This illustrates the scale of the problem: the integrand is of order one (at small $s$ ) and only known via numerical estimators for a realistic theory. The result, i.e., $Z$, is typically suppressed by many orders of magnitude for large volumes $V$. To achieve an acceptable signal to noise ratio, it is a prerequisite for success that the density-of-states is obtained over many orders of magnitude. This is what the LLR method can deliver. Despite of the exponential error suppression inherent to the LLR approach (see 2.3), it is not clear if the achieved precision for $P_{\beta}(s)$ is high enough to give meaning full results after Fourier transform. 

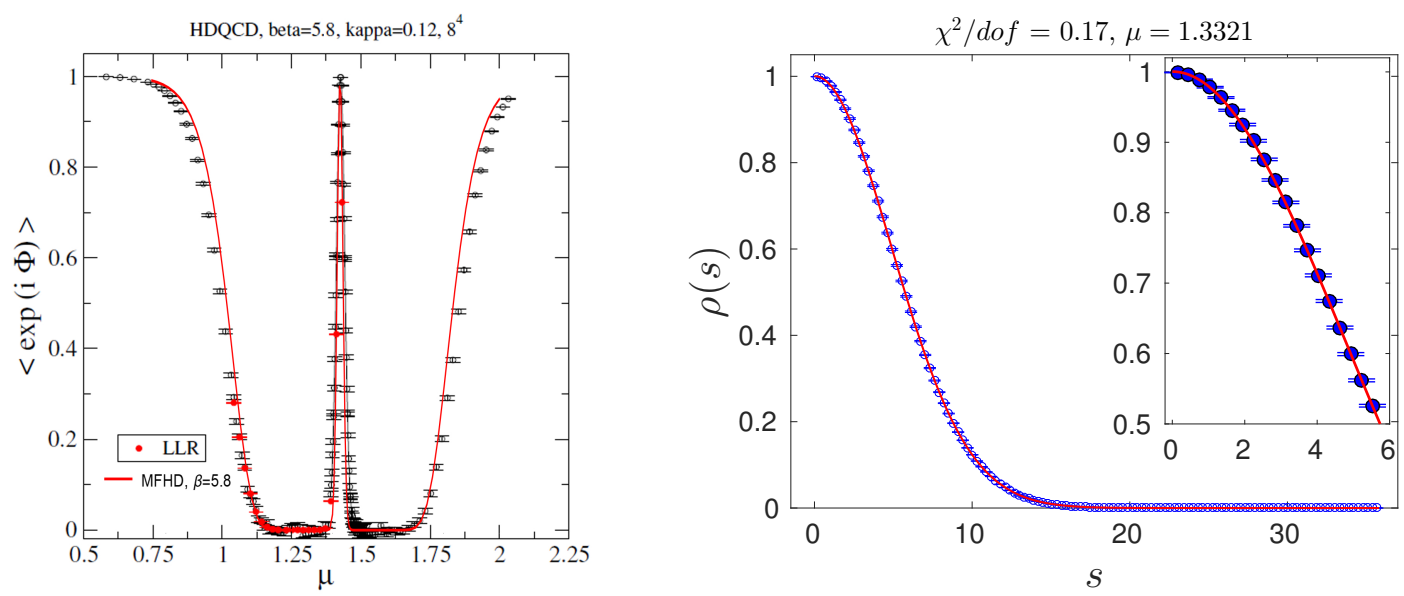

Figure 6: Left: The phase factor expectation value using re-weighting on the basis of a phase-quenched simulation (black symbols) and the mean-field result by Rindlisbacher and de Forcrand. Right: Probability distribution of the phase for $\mu=1.3321$ and the corresponding polynomial fit.

\subsection{Fourier transform of the generalised density-of-states}

Using the $Z_{3}$ spin model as showcase, Lucini and myself were able to show that good results can be achieved by a Fourier transformation of the generalised density-of-states [22]. The method that we put forward was:

(i) Perform a polynomial fit of the logarithm of the density-of-states with $p$ the degree of the polynomial: $\ln P_{\beta}(s)=\sum_{i}^{p} c_{i} s^{i}$.

(ii) Calculate the Fourier transform of $\exp \left\{\sum_{i}^{p} c_{i} s^{i}\right\}$ semi-analytical.

(iii) Increase $p$ and check for stability.

Recently, Garron and myself also applied the method in the context of QCD at finite densities of heavy quarks, the so called HDQCD (see [23]). Starting point is the QCD partition function

$$
Z(\mu)=\int \mathscr{D} U_{\mu} \exp \left\{\beta S_{\mathrm{YM}}[U]\right\} \operatorname{Det} M(\mu),
$$

where $M(\mu)$ is the quark operator. In the so-called heavy dense limit [24, 25] of both, large quark mass $m$ and large chemical potential $\mu$ with a finite ratio of $\mu / m$, the quark determinant factorises in a product of local determinants. This feature greatly simplifies the numerical approach leaving HDQCD as an ideal testbed for methods dealing with sign problems (see e.g. [26]). A standard MC simulation of the phase quenched theory and re-weighting with respect to the phase factor of the quark determinant provides a first glimpse on the severeness of the sign problem. Our reweighting result is shown as a function of the chemical potential in figure 6 in comparison with that from a mean-field approach $[27,28]^{3}$. HDQCD possesses a variety of interesting features: in the large mass limit, the graph exhibits a particle-duality and therefore a symmetry around $\mu=m$. The theory is a also real at the threshold $\mu=m$ (or at so-called half-filling: at threshold, the density is half of the saturation density at large $\mu$ ). Of high importance here is that we encounter a strong sign problem close to threshold $\mu<m$ where the re-weighting approach fails to produce

\footnotetext{
${ }^{3}$ I am grateful to Tobias Rindlisbacher and Philippe de Forcrand for providing the mean field result.
} 
reliable data. Using $\mu=1.3321$, which falls into the strong-sign problem regime, figure 6 also shows the generalised probability distribution $P_{\beta}(s)$ where $s$ is the sum of all the phases of the local determinants (see [23] for details). Also shown in this graph is the polynomial fit. As for the $Z_{3}$ theory [22], we empirically observe the importance of data compression: whenever we succeeded to represent hundreds of numerical data points of $\ln P_{\beta}(s)$ by a few (e.g. of order 7) fitting coefficients, the subsequent semi-analytical Fourier transformation produces reliable and significant results with acceptable size of the bootstrap errors. For HDQCD, figure 7 shows our final result for the logarithm of the phase factor expectation value as a function of the chemical potential. Bootstrap error bars are within the plotting symbols.
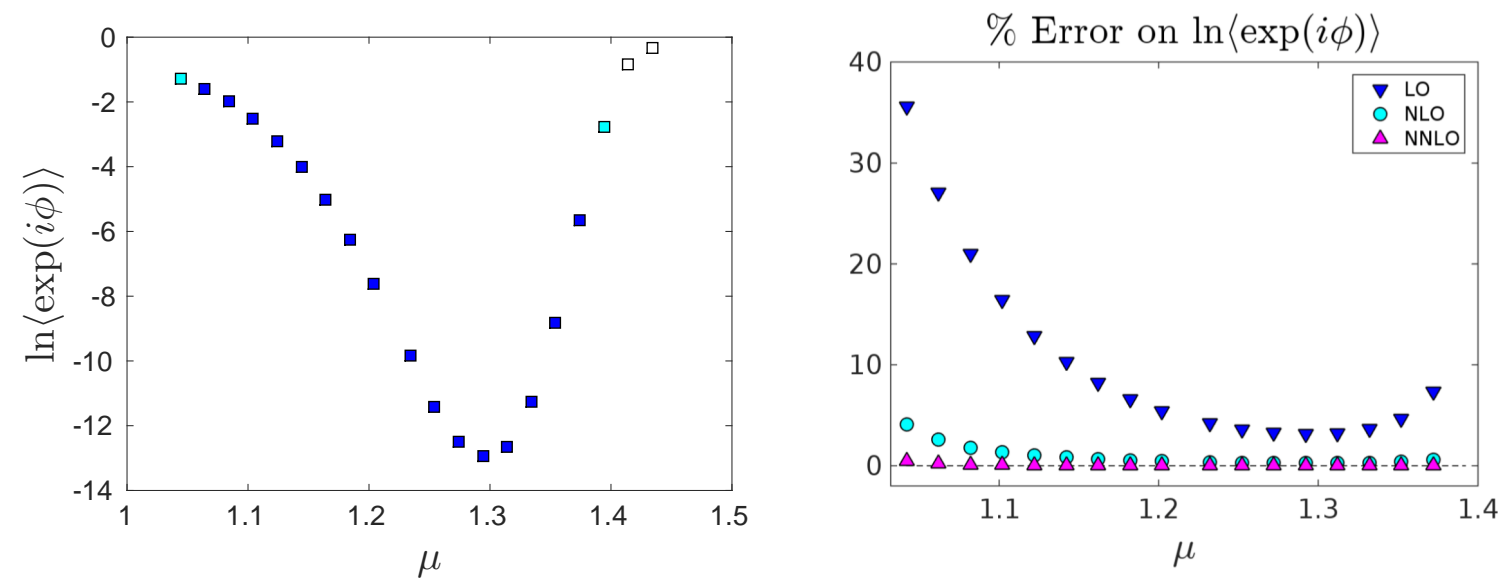

Figure 7: Left: The phase factor expectation value using the LLR method, polynomial fit and semi-analytic Fourier transformation (as outlined in the text). Right: relative error of the extended cumulant expansion over the whole $\mu$ range.

\subsection{Extended cumulant expansion for complex action systems}

The success of the method described in the previous subsection hinges on the need to find a fit function that represents the multitude of data with relatively few fit parameters. This approach was successful for the $Z_{3}$ theory and HDQCD for the strong-sign problem regime, but might fail if such a fit function cannot be found. Here, we wish to propose an alternative more systematic approach to the Fourier transform of the density-of-states. If $\phi \in]-\pi, \pi]$ denotes the phase of the quark determinant, it is well known $[29,30]$ that the probability distribution (or generalised density-ofstates) is almost uniform in the strong sign problem regime and that deviations of the Gaussian distributions are suppressed by powers of the volume $V$ :

$$
P(\phi)=1+\varepsilon a_{1} \phi^{2}+\varepsilon^{3} a_{2} \phi^{4}+\varepsilon^{6} a_{3} \phi^{6} \ldots, \quad \varepsilon=1 / V,
$$

where (for large volumes) the coefficient $a_{i}$ are volume independent and non-zero. As usual, the phase factor expectation value is given by Fourier transform:

$$
\left\langle\mathrm{e}^{i \phi}\right\rangle=\frac{1}{N} \int_{-\pi}^{\pi} d \phi P(\phi) \mathrm{e}^{i \phi}, \quad N=\int_{-\pi}^{\pi} d \phi P(\phi) .
$$


Our method [31] performs a systematic expansion of $\left\langle\mathrm{e}^{i \phi}\right\rangle$ with respect to $\varepsilon$. The zeroth order $P(\phi)=1$ trivially yields $\left\langle\mathrm{e}^{i \phi}\right\rangle=0$, which is good starting point for the strong sign-problem regime. Using the standard moments, i.e., $\left\langle\phi^{n}\right\rangle=\frac{1}{N} \int_{-\pi}^{\pi} \phi^{n} P(\phi) d \phi$, we define the extended cumulants by

$$
\begin{aligned}
& M_{4}=\left\langle\phi^{4}\right\rangle-\frac{3 \pi^{2}}{5}\left\langle\phi^{2}\right\rangle=\mathscr{O}(\varepsilon), \quad M_{6}=\left\langle\phi^{6}\right\rangle-\frac{10 \pi^{2}}{9}\left\langle\phi^{4}\right\rangle+\frac{5 \pi^{4}}{21}\left\langle\phi^{2}\right\rangle=\mathscr{O}\left(\varepsilon^{3}\right), \\
& M_{8}=\left\langle\phi^{8}\right\rangle-\frac{21 \pi^{2}}{13}\left\langle\phi^{6}\right\rangle+\frac{105 \pi^{4}}{143}\left\langle\phi^{4}\right\rangle-\frac{35 \pi^{6}}{429}\left\langle\phi^{2}\right\rangle=\mathscr{O}\left(\varepsilon^{6}\right) .
\end{aligned}
$$

The systematic expansion of the phase factor expectation value then can be facilitated by means of these moments:

$$
\left\langle\mathrm{e}^{i \phi}\right\rangle=-\frac{175}{2 \pi^{6}} M_{4}+\frac{4851\left(27-2 \pi^{2}\right)}{8 \pi^{10}} M_{6}-\frac{57915\left(3 \pi^{4}-242 \pi^{2}+2145\right)}{16 \pi^{14}} M_{8}+\mathscr{O}\left(\varepsilon^{8}\right) .
$$

Garron and myself have tested the moment expansion in the context of HDQCD. Choosing $\mu=$ 1.2921 from the strong sign-problem regime, we find order by order:

$$
\left\langle\mathrm{e}^{i \phi}\right\rangle=10^{-6}[1.45(28)+0.67(13)+0.068(13)]+\mathscr{O}\left(\varepsilon^{8}\right)
$$

Figure 7, right panel, shows the relative error of the result of the extended cumulant expansion as a function of $\mu$ in relation to the "exact" results from the previous subsection. A expected, the expansion works best in the strong sign-problem regime where the deviation of $P(\phi)$ from uniformity are smallest. Note, however, that even at small $\mu$, the method yields remarkable results.

\section{Conclusions}

Importance sampling Monte-Carlo based upon local updates fails for systems near first order transitions due to a suppression of tunneling between states of equal importance. They also fail if the Gibbs factor is not (semi-) positive definite, which excludes the large class of quantum field theories at finite matter densities. Cluster algorithms provide a solution if one succeeds to identify and update physically relevant clusters. The definition of physical clusters might also emerge from dualisation, and the dual theory might be real solving the sign problem in this case as well $[8,32,4]$.

Rather than devising global update strategies, we can retain local updates and abandon Importance Sampling. Non-Markovian Random Walks propose updates with a self-improving estimate of the density-of-states, thus incorporating memory, until a random walk in configuration space is reached. This approach bears the potential to overcome the ergodicity problems near first order transitions. An important attempt is this direction is the multicanonical algorithm [9, 19]. However, this algorithm attempts an update of the density-of-states over the whole action range at a time, and the initial guess of this density severely limits the performance of the algorithm near criticality. Significant progress is provided by the Wang-Landau type techniques [10]: while the update is again informed by the density-of-states, updates are limited to action windows, and results are collated once a random walk is achieved in each action window. Note that at this stage, the overlap problem has been avoided. The LLR method that has been extensively discussed in this paper belongs to this class. While the standard Wang-Landau algorithms are based upon action histograms, the LLR 
method is based upon moments of the probability distribution avoiding histograms at all. This has advantages for theories with continuous degrees of freedom.

Subsequent to the first publication on LLR in 2012 [11], concerns have been raised in relation to the action window impeding ergodicity and the range of applications. Excellent progress has been made over the recent years and some of it has been summarised in his paper:

- Using a Replica Exchange within the LLR iteration (see subsection 2.5) guarantees conceptual ergodicity. Moreover at a practical level, we have not observed any critical slowing down or ergodicity issues for the $q=20$ state Potts model (see also [33]). A systematic study of the volume scaling of thermodynamical quantities at criticality is left to future work.

- Using a smooth window function (see subsection 2.6) featuring in LLR expectation values allows the design of very efficient algorithms (see also [34]). On the basis of LHMC, results for the latent heat of a pure SU(3) Yang-Mills theory at criticality have been firstly reported here (see subsection 2.6, see also [35]).

- The LLR approach can be used to calculate any observables alongside the density-of-states not just action based observables. The theoretical framework for this has been presented in [12]. Note also that the approach can be easily generalised to calculate observables with unprecedented precision. This has been e.g. achieved for the SU(2) Polyakov line [20].

- The LLR approach is not hampered by a complex action and is therefore a candiate algorithm to study finite density quantum field theory. Here, the numerical task is to extract a very small signal from the Fourier transform of the (generalised) density-of-states (see section 3). The LLR algorithm features the remarkable property of an exponential error suppression, and it has been shown that at least for some theories a solution of sign problem in the theory's original formulation is indeed feasible [22, 23].

Non-Markovian Random walks as the Wang-Landau type methods such as the LLR algorithm might provide the key to overcome critical slowing down inherent to theories near first order criticality. More important, however, is that the generalised LLR approach to complex action systems is one of the rare methods available today that are exact, do not rely on a specific property of the theory and that deliver controllable errors all at the same time.

Acknowledgements: It is pleasure to thank my collaborators on LLR projects: L. Bongiovanni, Ch. Gattringer, N. Garron, B. Lucini, J. Pawlowski, R. Pellegrini, A. Rago.

\section{References}

[1] S. Schaefer et al. [ALPHA Collaboration], Nucl. Phys. B 845 (2011) 93 [arXiv:1009.5228 [hep-lat]].

[2] S. Borsanyi, PoS LATTICE 2015 (2016) 015 [arXiv:1511.06541 [hep-lat]].

[3] D. Sexty, PoS LATTICE 2014 (2014) 016 [arXiv:1410.8813 [hep-lat]].

[4] C. Gattringer, PoS LATTICE 2013 (2014) 002 [arXiv:1401.7788 [hep-lat]].

[5] G. Aarts, PoS LATTICE 2012 (2012) 017 [arXiv:1302.3028 [hep-lat]]. 
[6] U. Wolff, PoS LATTICE 2010 (2010) 020 [arXiv:1009.0657 [hep-lat]].

[7] P. de Forcrand, PoS LAT 2009 (2009) 010 [arXiv:1005.0539 [hep-lat]].

[8] S. Chandrasekharan, PoS LATTICE 2008 (2008) 003 [arXiv:0810.2419 [hep-lat]].

[9] B. A. Berg and T. Neuhaus, Phys. Rev. Lett. 68 (1992) 9 [hep-lat/9202004].

[10] F. Wang and D. P. Landau, Phys. Rev. Lett. 86 (2001) 2050 [arXiv:cond-mat/0011174]

[11] K. Langfeld, B. Lucini and A. Rago, Phys. Rev. Lett. 109 (2012) 111601 [arXiv:1204.3243 [hep-lat]].

[12] K. Langfeld, B. Lucini, R. Pellegrini and A. Rago, Eur. Phys. J. C 76 (2016) no.6, 306 [arXiv:1509.08391 [hep-lat]].

[13] C. Gattringer and K. Langfeld, Int. J. Mod. Phys. A 31 (2016) no.22, 1643007 [arXiv:1603.09517 [hep-lat]].

[14] C. Gattringer and P. Törek, Phys. Lett. B 747 (2015) 545 [arXiv:1503.04947 [hep-lat]].

[15] M. Giuliani, C. Gattringer and P. Törek, Nucl. Phys. B 913 (2016) 627 [arXiv:1607.07340 [hep-lat]].

[16] H. Robbins, S. Monro, The Annals of Mathematical Statistics 22 (1951) 400.

[17] Robert H. Swendsen and Jian-Sheng Wang, Phys. Rev. Lett. 57 (1986) 2607

[18] R. J. Baxter, J. Phys. C 6 (1973) L445.

[19] A. Billoire, T. Neuhaus and B. A. Berg, Nucl. Phys. B 413 (1994) 795 [hep-lat/9307017].

[20] K. Langfeld and J. M. Pawlowski, Phys. Rev. D 88 (2013) no.7, 071502 [arXiv:1307.0455 [hep-lat]].

[21] A. Gocksch, Phys. Rev. Lett. 61 (1988) 2054.

[22] K. Langfeld and B. Lucini, Phys. Rev. D 90 (2014) no.9, 094502 [arXiv:1404.7187 [hep-lat]].

[23] N. Garron and K. Langfeld, Eur. Phys. J. C 76 (2016) no.10, 569 [arXiv:1605.02709 [hep-lat]].

[24] I. Bender, T. Hashimoto, F. Karsch, V. Linke, A. Nakamura, M. Plewnia, I. O. Stamatescu and W. Wetzel, Nucl. Phys. Proc. Suppl. 26 (1992) 323.

[25] T. C. Blum, J. E. Hetrick and D. Toussaint, Phys. Rev. Lett. 76 (1996) 1019 [hep-lat/9509002].

[26] G. Aarts, F. Attanasio, B. Jäger and D. Sexty, JHEP 1609 (2016) 087 [arXiv:1606.05561 [hep-lat]].

[27] T. Rindlisbacher and P. de Forcrand, JHEP 1602 (2016) 051 [arXiv:1509.00087 [hep-lat]].

[28] O. Akerlund, P. de Forcrand and T. Rindlisbacher, JHEP 1610 (2016) 055 [arXiv:1602.02925 [hep-lat]].

[29] H. Saito, S. Ejiri, S. Aoki, K. Kanaya, Y. Nakagawa, H. Ohno, K. Okuno and T. Umeda, Phys. Rev. D 89 (2014) no.3, 034507 [arXiv:1309.2445 [hep-lat]].

[30] J. Greensite, J. C. Myers and K. Splittorff, JHEP 1310 (2013) 192 [arXiv:1308.6712 [hep-lat]].

[31] N. Garron and K. Langfeld, Tackling the sign problem with a moment expansion and application to Heavy dense QCD, arXiv:1611.01378 [hep-lat].

[32] K. Langfeld, Phys. Rev. D 87 (2013) no.11, 114504 [arXiv:1302.1908 [hep-lat]].

[33] B. Lucini, K. Langfeld, Lattice 2016, Overcoming strong metastabilities with the LLR method.

[34] R. Pellegrini, V. Davide, B. Lucini, A. Rago, Lattice 2016, Computing the density of states with the global Hybrid Monte Carlo.

[35] Shinji Ejiri, for the WHOT collaboration, Lattice 2016, Determination of latent heat at the finite temperature phase transition of $S U(3)$ gauge theory . 